\title{
OPTIMAL BACKWARD AND BACKUP POLICIES IN RELIABILITY THEORY
}

\author{
Mingchih Chen \\ Chaoyang University of Technology
}

\author{
Satoshi Mizutani \\ Aichi University of Technology
}

\author{
Toshio Nakagawa \\ Aichi Institute of Technology
}

(Received June 17, 2009; Revised October 30, 2009)

\begin{abstract}
We are sometimes faced with the problem of searching for the actual failure time of a unit when it has failed until time $t_{0}$. This is called a backward time problem of how much time we go back to search for the failure time from time $t_{0}$, and is solved by using the reversed failure rate. This paper proposes an extended model of multiple backward trials in which we find the actual failure time from time $t_{0}$. Another problem is that when an operating unit fails, we have to go back to the newest checking time and reconstruct it as recovery techniques, which is called a backup policy. This paper takes up three backup policies where the unit is checked at periodic, successive and random working times, and discusses analytically their optimal policies. Finally, it would be important to keep a record of operational behavior of a unit when its failure is detected, which is called traceability. This paper compares two models with traceability and without traceability, and proposes three traceability models where the unit operates for a finite interval, and is checked at periodic and successive times. Optimal policies for each model are discussed by using backup techniques.
\end{abstract}

Keywords: Reliability, backward time, backup policy, reversed failure rate, traceability

\section{Introduction}

The first problem in reliability theory is to estimate when an objective unit will fail in the near future. From such viewpoints, data concerning failures are collected as possible as one can, and failure distributions and their parameters have been estimated statistically. Using the fitted failure distributions, some reliability measures have been well designed, and maintenance policies to prevent failures based on them have been discussed practically and analytically in Barlow and Proschan [1], Nakagawa [7,9], Osaki [15] and Pham [16].

The second problem is how to find a failure of the unit with certainty which can not be detected immediately at failure. This leads to inspection policies where the unit is checked at suitable times to detect its failure summarized in Barlow and Proschan $[1$, p. 107] and Nakagawa [7, p. 20].

The third problem is how to know the actual failure time when we detect a failed unit. This was applied to the problem of reweighing products using a scale in Sandoh et al. $[19,20]$. Another example is the backup policy for a database system in Naruse et al. [14]. When a failure occurs in the operation of a database system, we execute the rollback operation until the newest checkpoint, recover the data and files and reconstruct the system. A variety of checkpoint schemes for some database systems has been actually carried out and has been compared practically and analytically in Fukumoto et al. [4], Nakagawa et al. [10], Nakagawa [9, p. 123] and Reuter [18]. Furthermore, some backup policies such as incremental, cumulative, full and complete backups introducing in Velpuri and Adkoli [22] as recovery techniques have been adopted for a database system, and their optimal policies 
have been extensively discussed in Nakamura et al. [13] and Qian et al. [17] by applying cumulative damage models summarized in Nakagawa [8].

Our main purpose in this paper is to survey some backward models in Nakagawa and Mizutani [12] and backup policies in Naruse et al. [14], and add some new interesting results by using reliability theory: In Section 2, we consider the optimization problem of how much time we go back to search for the actual failure time, which is called a backward time. Introducing two costs of excess and shortage times, we derive analytically an optimal backward time which minimizes the expected cost. It is of interest theoretically that the reversed failure rate studied by Chandra and Roy [2] and Finkelstein [3] plays an important role in analyzing the optimal policy. Furthermore, we extend the backward time to multiple backward times: When we cannot find a failure time at one trial, we attempt the next trial. Such trials are continued until failure detection. Successive backward times are derived by using the inspection policy for a finite interval in Nakagawa and Mizutani [11].

In Section 3, we consider the backup policy where the unit goes back to the last checking time when it fails, and formulate its three stochastic models: The unit is checked at periodic times, at successive times and at random working times. Three models are analyzed by using the methods of backup of Naruse et al. [14], inspection of Nakagawa [7, p. 201] and random maintenance of Nakagawa [7, p. 245].

Finally, when the unit is detected to have failed, it would be much easier to know its actual failure time if its operational behaviors have been on record. This is called the traceability and is commonly used in food products and sales studies in Lees [6]. In Section 4, we compare two models with traceability and without traceability. Next, we propose three traceability models where the unit operates for a finite time interval, and is checked at periodic and successive times. Three models are analyzed and their optimal policies are derived by using maintenance theory.

\section{Backward Models}

Suppose that the unit begins to operate at time 0 and has a failure time $X$ with its probability distribution $F(t) \equiv \operatorname{Pr}\{X \leq t\}$, a density function $f(t) \equiv \mathrm{d} F(t) / \mathrm{d} t$ and a mean time $\mu \equiv \int_{0}^{\infty} \bar{F}(t) \mathrm{d} t<\infty$, where $\bar{\Phi}(t) \equiv 1-\Phi(t)$ for any function $\Phi(t)$. Then, the probability that the unit failed during $\left(t_{0}-x, t_{0}\right]\left(0 \leq x \leq t_{0}\right)$, given that it is detected to have failed at time $t_{0}\left(0<t_{0}<\infty\right)$ is

$$
H\left(x \mid t_{0}\right) \equiv \operatorname{Pr}\left\{t_{0}-x \leq X \mid X \leq t_{0}\right\}=\frac{F\left(t_{0}\right)-F\left(t_{0}-x\right)}{F\left(t_{0}\right)} \leq 1
$$

for $F\left(t_{0}\right)>0$, which is called the reversed failure rate. The properties of $H\left(x \mid t_{0}\right)$ were investigated in Chandra and Roy [2] and Finkelstein [3].

\subsection{Continuous time}

Consider the optimization problem of how much time we go back to catch the actual failure time: Suppose that when the unit is detected to have failed at time $t_{0}\left(0<t_{0}<\infty\right)$ and its failure time is unknown, we go back time $T\left(0 \leq T \leq t_{0}\right)$ from time $t_{0}$ to detect its failure time, where $T$ is called the planned backward time.

Next, introduce the following loss costs (Figure 1): Cost $c_{1}(x)$ is the excess cost for the time $x$ from a failure to the backward time, $c_{2}(x)$ is the shortage cost for the time $x$ from the backward time to a failure, and $c_{3}(T)$ is the cost required for the backward time $T$. Cost $c_{3}(T)$ is irrelevant to the system condition although the failure has occurred, and is the cost only related to the time interval $T$. Consequently, we need to introduce the other 


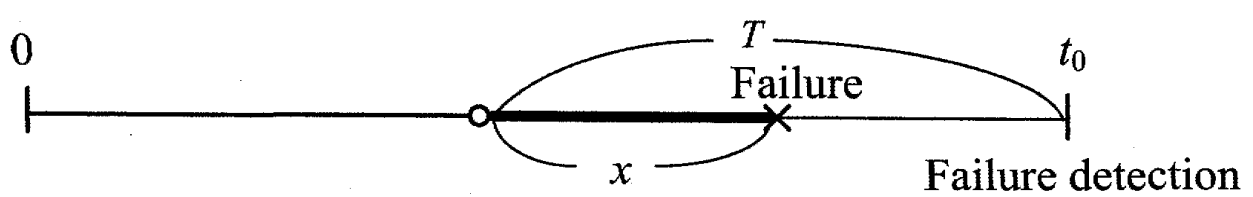

Excess cost $c_{1}(x)$

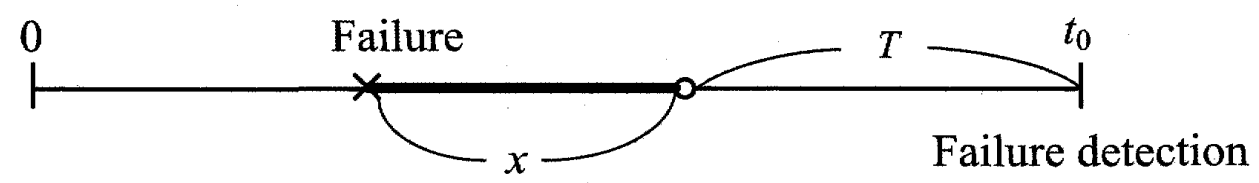

Shortage $\operatorname{cost} c_{2}(x)$

Figure 1: Excess cost and shortage cost of backward time $T$

two costs $c_{1}(x)$ and $c_{2}(x)$ which represent all corresponding costs for the backward models. If $c_{1}(x)$ would be included in $c_{3}(T)$, it might be assumed to be zero. One typical example of the backward model is such as automobile companies recalled their cars for some defective parts. The cost $c_{3}(T)$ represents the cost for how many years to recall back, e.g., $T=3$ means to recall the cars which were sold in recent 3 years. However, if the defective cars happened only for the recent 2 years, the company might need to pay excess cost for the extra parts ordered for the cars which are good. If the actual defective cars were sold for 4 years, cost $c_{2}(x)$ is used to described such situation. is

Using the definition of $H\left(x \mid t_{0}\right)$ in (1), the total expected cost for the backward time $T$

$$
\begin{aligned}
C_{1}\left(T \mid t_{0}\right) & =\int_{0}^{T} c_{1}(T-x) \mathrm{d} H\left(x \mid t_{0}\right)+\int_{T}^{t_{0}} c_{2}(x-T) \mathrm{d} H\left(x \mid t_{0}\right)+c_{3}(T) \\
& =\frac{1}{F\left(t_{0}\right)}\left[\int_{t_{0}-T}^{t_{0}} c_{1}\left(x-t_{0}+T\right) \mathrm{d} F(x)+\int_{0}^{t_{0}-T} c_{2}\left(t_{0}-T-x\right) \mathrm{d} F(x)\right]+c_{3}(T)
\end{aligned}
$$

In particular, suppose that $H\left(x \mid t_{0}\right)=\left(x / t_{0}\right)^{\beta}$ for $\beta>0$ and $0 \leq x \leq t_{0}$, which is wellknown as a uniform distribution when $\beta=1$ and a triangular distribution when $\beta=2$. In this case, the total expected cost is, from (2),

$$
C_{1}\left(T \mid t_{0}\right)=\frac{\beta}{t_{0}}\left[\int_{0}^{T} c_{1}(x)\left(\frac{T-x}{t_{0}}\right)^{\beta-1} \mathrm{~d} x+\int_{0}^{t_{0}-T} c_{2}(x)\left(\frac{T+x}{t_{0}}\right)^{\beta-1} \mathrm{~d} x\right]+c_{3}(T)
$$

In case of $\beta=1$,

$$
C_{1}\left(T \mid t_{0}\right)=\frac{1}{t_{0}}\left[\int_{0}^{T} c_{1}(x) \mathrm{d} x+\int_{0}^{t_{0}-T} c_{2}(x) \mathrm{d} x\right]+c_{3}(T)
$$

It is assumed that $c_{i}(t)=c_{i} t(i=1,2,3)$. Then, the total expected cost in (2) is

$$
C_{1}\left(T \mid t_{0}\right)=\frac{1}{F\left(t_{0}\right)}\left[-c_{1} \int_{t_{0}-T}^{t_{0}} F(x) \mathrm{d} x+c_{2} \int_{0}^{t_{0}-T} F(x) \mathrm{d} x\right]+\left(c_{1}+c_{3}\right) T .
$$


Table 1: Optimal time $T^{*}$ when $c_{3} / c_{1}=0.5$

\begin{tabular}{|c|c|c|c|c|c|c|c|c|c|}
\hline \multirow{3}{*}{$t_{0}$} & \multicolumn{3}{|c|}{$c_{2} / c_{1}=1$} & \multicolumn{3}{|c|}{$c_{2} / c_{1}=2$} & \multicolumn{3}{|c|}{$c_{2} / c_{1}=5$} \\
\hline & & $\beta$ & & & $\beta$ & & & $\bar{\beta}$ & \\
\hline & 1 & 2 & 4 & 1 & 2 & 4 & 1 & 2 & 4 \\
\hline 10 & 2.50 & 5.00 & 7.07 & 5.00 & 7.07 & 8.41 & 7.50 & 8.66 & 9.31 \\
\hline 20 & 5.00 & 10.00 & 14.14 & 10.00 & 14.14 & 16.82 & 15.00 & 17.32 & 18.61 \\
\hline 50 & 12.50 & 25.00 & 35.36 & 25.00 & 35.36 & 42.05 & 37.50 & 43.30 & 46.53 \\
\hline 100 & 25.00 & 50.00 & 70.71 & 50.00 & 70.71 & 84.10 & 75.00 & 86.60 & 93.06 \\
\hline 200 & 50.00 & 100.00 & 141.42 & 100.00 & 141.42 & 68.20 & 150.00 & 173.21 & 186.12 \\
\hline 500 & 125.00 & 250.00 & 353.55 & 250.00 & 353.55 & 420.49 & 375.00 & 433.01 & 465.30 \\
\hline
\end{tabular}

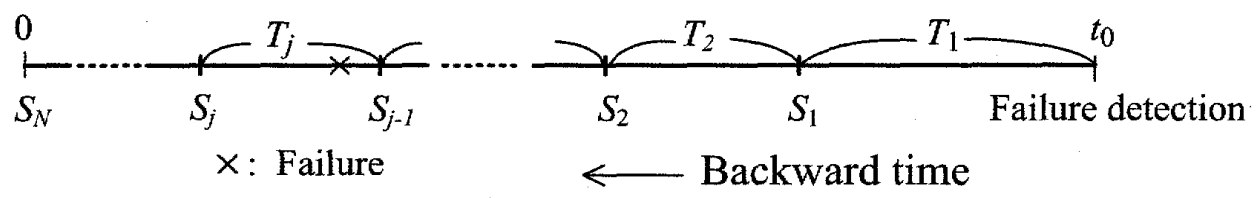

Figure 2: Process of multiple backward times

We find an optimal backward time $T^{*}$ which minimizes $C_{1}\left(T \mid t_{0}\right)$ for a specified $t_{0}>0$. Differentiating $C_{1}\left(T \mid t_{0}\right)$ with respect to $T$ and setting it equal to zero,

$$
H\left(T \mid t_{0}\right)=\frac{F\left(t_{0}\right)-F\left(t_{0}-T\right)}{F\left(t_{0}\right)}=\frac{c_{2}-c_{3}}{c_{2}+c_{1}} .
$$

Therefore, we have the following optimal policy:

(i) If $c_{2}>c_{3}$ then there exists a unique $T^{*}\left(0<T^{*}<t_{0}\right)$ which satisfies (6).

(ii) If $c_{2} \leq c_{3}$ then $T^{*}=0$, i.e., we should not go back at all.

Condition of $\left\{c_{2} \leq c_{3}\right\}$ means the trivial result that it is not necessary to search for the failure time if the shortage cost is equal to or lower than that for backward.

In particular, when $H\left(x \mid t_{0}\right)=\left(x / t_{0}\right)^{\beta}$, an optimal time $T^{*}$ is, for $c_{2}>c_{3}$,

$$
T^{*}=\left(\frac{c_{2}-c_{3}}{c_{2}+c_{1}}\right)^{1 / \beta} t_{0}
$$

Table 1 presents optimal times $T^{*}$ for $t_{0}=10 \sim 500, c_{2} / c_{1}=1,2,5$ and $\beta=1,2,4$ when $c_{3} / c_{1}=0.5$. For example, this indicates that when the failure is detected at time $t_{0}$, $c_{2} / c_{1}=1$ and $\beta=2$ or $c_{2} / c_{1}=2$ and $\beta=1$, we may go back its half interval. It can be clearly seen that optimal $T^{*}$ increase with $t_{0}, c_{2} / c_{1}$ and $\beta$.

\subsection{Multiple times}

Consider the multiple backward times $T_{j}(j=1,2, \ldots)$ (Figure 2), where $S_{j} \equiv \sum_{i=1}^{j} T_{i}, S_{0} \equiv$ 0 and $S_{N} \equiv t_{0}$ : If we cannot find the actual failure time until time $S_{j-1}$ from time $t_{0}$ by the backward operation, we execute again the next backward with time $T_{j}$. If we can find the failure time, i.e., the system fails in $\left(t_{0}-S_{j}, t_{0}-S_{j-1}\right]$, then the process ends. Finally, if we cannot find the failure time until $S_{N-1}$, we make the final backward with $T_{N}=t_{0}-S_{N-1}$, and can find it certainly.

It is assumed that $c_{1}+c_{2} T_{j}$ is the cost for the $j$ th backward. Then, because the expected 
cost when the failure time is detected at the $j$ th backward is

$$
\frac{1}{F\left(t_{0}\right)}\left(c_{1} j+c_{2} S_{j}\right)\left[F\left(t_{0}-S_{j-1}\right)-F\left(t_{0}-S_{j}\right)\right]
$$

the total expected cost is

$$
C_{2}(\mathbf{T})=\frac{1}{F\left(t_{0}\right)} \sum_{j=1}^{N}\left(c_{1} j+c_{2} S_{j}\right)\left[F\left(t_{0}-S_{j-1}\right)-F\left(t_{0}-S_{j}\right)\right] .
$$

We find optimal times $T_{j}(j=1,2, \ldots, N)$ which minimize $C_{2}(\mathrm{~T})$ for a given $t_{0}>0$. Differentiating $C_{2}(\mathbf{T})$ with respect to $S_{j}$ and setting it equal to zero,

$$
S_{j+1}-S_{j}=\frac{F\left(t_{0}-S_{j-1}\right)-F\left(t_{0}-S_{j}\right)}{f\left(t_{0}-S_{j}\right)}-\frac{c_{1}}{c_{2}} \quad(j=1,2, \ldots, N-1) .
$$

Setting that $x_{j} \equiv t_{0}-S_{j}$,

$$
x_{j}-x_{j+1}=\frac{F\left(x_{j-1}\right)-F\left(x_{j}\right)}{f\left(x_{j}\right)}-\frac{c_{1}}{c_{2}} \quad(j=1,2, \ldots, N-1),
$$

where $x_{N} \equiv 0$ and $x_{0} \equiv t_{0}$. Then, the total expected cost is

$$
C_{2}(\mathbf{x})=\frac{1}{F\left(t_{0}\right)} \sum_{j=1}^{N}\left[c_{1} j+c_{2}\left(t_{0}-x_{j}\right)\right]\left[F\left(x_{j-1}\right)-F\left(x_{j}\right)\right]
$$

We compute optimal $0=x_{N}<x_{N-1}<\cdots<x_{1}<t_{0}$ which satisfy (10) for a given $N(N=1,2, \ldots)$. Next, comparing $C_{2}(\mathbf{x})$ in (11) for all $N \geq 1$, we can get an optimal backward number $N^{*}, x_{j}^{*}$ and $T_{j}^{*}=x_{j-1}^{*}-x_{j}^{*}=S_{j}^{*}-S_{j-1}^{*}$. For example, when $N=1$,

$$
\begin{gathered}
x_{1}=0, T_{1}=t_{0}, \\
C_{2}(0)=c_{1}+c_{2} t_{0} .
\end{gathered}
$$

When $N=2$, from (10) and (11),

$$
\begin{aligned}
x_{1} & =\frac{F\left(t_{0}\right)-F\left(x_{1}\right)}{f\left(x_{1}\right)}-\frac{c_{1}}{c_{2}} \\
C_{2}\left(x_{1}\right) & =c_{1}+c_{2}\left(t_{0}-x_{1}\right)+\frac{F\left(x_{1}\right)}{F\left(t_{0}\right)}\left(c_{1}+c_{2} x_{1}\right) .
\end{aligned}
$$

When $N=3$, we solve the simultaneous equations

$$
\begin{aligned}
x_{1}-x_{2} & =\frac{F\left(t_{0}\right)-F\left(x_{1}\right)}{f\left(x_{1}\right)}-\frac{c_{1}}{c_{2}}, \\
x_{2} & =\frac{F\left(x_{1}\right)-F\left(x_{2}\right)}{f\left(x_{2}\right)}-\frac{c_{1}}{c_{2}},
\end{aligned}
$$

and compute the total expected cost

$$
C_{2}\left(x_{1}, x_{2}\right)=c_{1}+c_{2}\left(t_{0}-x_{1}\right)+\frac{1}{F\left(t_{0}\right)}\left\{F\left(x_{1}\right)\left[c_{1}+c_{2}\left(x_{1}-x_{2}\right)\right]+F\left(x_{2}\right)\left(c_{1}+c_{2} x_{2}\right)\right\}
$$


Table 2: Maximum number $\widetilde{N}$

\begin{tabular}{c|cccc}
\hline & \multicolumn{4}{|c}{$c_{2} /\left(\lambda c_{1}\right)$} \\
\cline { 2 - 5 }$\lambda t_{0}$ & 1 & 2 & 5 & 10 \\
\hline 0.1 & 1 & 1 & 1 & 1 \\
0.2 & 1 & 1 & 1 & 2 \\
0.5 & 1 & 1 & 2 & 3 \\
1.0 & 1 & 2 & 3 & 4 \\
1.5 & 2 & 2 & 4 & 5 \\
2.0 & 2 & 3 & 4 & 6 \\
\hline
\end{tabular}

We compute similar computations for $N=4,5, \ldots$.

In particular, when $F(t)=1-\mathrm{e}^{-\lambda t},(9)$ is

$$
\lambda T_{j+1}=1-\mathrm{e}^{-\lambda T_{j}}-\frac{\lambda c_{1}}{c_{2}} \quad(j=1,2, \ldots, N-1),
$$

and from (11),

$$
C_{2}(\mathrm{~T})=\frac{\mathrm{e}^{-\lambda t_{0}}}{1-\mathrm{e}^{-\lambda t_{0}}} \sum_{j=1}^{N}\left(j c_{1}+c_{2} \sum_{i=1}^{j} T_{i}\right) \mathrm{e}^{\lambda \sum_{i=1}^{j} T_{i}}\left(1-\mathrm{e}^{-\lambda T_{j}}\right) \quad(N=1,2, \ldots) .
$$

Because $\lambda T_{j}>1-\mathrm{e}^{-\lambda T_{j}},(12)$ becomes

$$
T_{j}-T_{j+1}>\frac{c_{1}}{c_{2}} \quad(j=1,2, \ldots, N-1) .
$$

Solving $T_{j}$ in terms of $T_{N}$,

$$
T_{j}-T_{N}>\frac{(N-j) c_{1}}{c_{2}}
$$

Noting that $\sum_{j=1}^{N} T_{j}=t_{0}$

$$
\frac{t_{0}}{N}>T_{N}+\frac{N-1}{2} \frac{c_{1}}{c_{2}}, \text { i.e., } T_{N}<\frac{t_{0}}{N}-\frac{N-1}{2} \frac{c_{1}}{c_{2}} .
$$

For $T_{N}>0$

$$
\frac{t_{0}}{N}>\frac{N-1}{2} \frac{c_{1}}{c_{2}}, \text { i.e., } \frac{N(N-1)}{2}=\sum_{j=1}^{N-1} j<\frac{t_{0} c_{2}}{c_{1}} .
$$

Therefore, the summation of $j$ from 1 to $N^{*}-1$ has to be less than $\left(\lambda t_{0}\right) c_{2} /\left(\lambda c_{1}\right)$. Table 2 presents the maximum number $\widetilde{N}$ which satisfies (15) for different $\lambda t_{0}$ and $c_{2} /\left(\lambda c_{1}\right)$. Table 3 presents the backward times $\lambda S_{j}$ for $j=1, \ldots, \widetilde{N}$ and $c_{2} /\left(\lambda c_{1}\right)=5,10$ when $\lambda t_{0}=1$. For example, when $c_{2} /\left(\lambda c_{1}\right)=10, \widetilde{N}=4>N^{*}=3$ and the optimal backward times are $\lambda T_{1}^{*}=0.526, \lambda T_{2}^{*}=0.308, \lambda T_{3}^{*}=0.166$. When $c_{2} /\left(\lambda c_{1}\right)=5, \widetilde{N}=3=N^{*}$, and the optimal backward times are $\lambda T_{1}^{*}=0.665, \lambda T_{2}^{*}=0.286, \lambda T_{3}^{*}=0.049$. This shows that the maximum number $\widetilde{N}$ can be easily computed from (15) and would be very useful for obtaining optimal backward number $N^{*}$ and backward times $T_{j}^{*}\left(j=1,2, \ldots, N^{*}\right)$. 
Table 3: Backward times $\lambda S_{j}$ and expected cost $C_{2}(\mathrm{~T}) / c_{1}$ when $\lambda t_{0}=1.0$

\begin{tabular}{c|cccc|ccc}
\hline & \multicolumn{4}{|c|}{$c_{2} /\left(\lambda c_{1}\right)=10$} & \multicolumn{3}{c}{$c_{2} /\left(\lambda c_{1}\right)=5$} \\
\cline { 2 - 8 }$j$ & \multicolumn{3}{|c}{$N$} & & \multicolumn{3}{c}{$N$} \\
\hline 1 & 1 & 2 & 3 & 4 & 1 & 2 & 3 \\
2 & & 0.632 & 0.526 & 0.504 & 1.0 & 0.698 & 0.665 \\
3 & & 1.0 & 0.834 & 0.800 & & 1.0 & 0.951 \\
4 & & & 1.0 & 0.956 & & & 1.0 \\
\hline$C_{2}(\mathbf{T}) / c_{1}$ & 11.00 & 9.60 & 9.34 & 9.90 & 6.00 & 5.53 & 5.51
\end{tabular}

Table 4: Expected cost $\widehat{C}_{2}(N) / c_{1}$ when $c_{2} /\left(\lambda c_{1}\right)=10$

\begin{tabular}{c|cc}
\hline$N$ & $\lambda t_{0}=1$ & $\lambda t_{0}=2$ \\
\hline 1 & 11.000 & 21.000 \\
2 & 9.600 & 18.669 \\
3 & 9.387 & 18.003 \\
4 & 9.513 & 17.862 \\
5 & 9.784 & 17.955 \\
\hline
\end{tabular}

\subsection{Approximation method}

It is very troublesome to solve simultaneous equations in (10). We consider the following approximate backward times using the approximation method of inspection policies in Kaio and Osaki [5] and Nakagawa [7, p. 20]: It is assumed that the probability with which the failure time is detected at the $j$ th backward is constant, i.e.,

$$
\frac{F\left(t_{0}-S_{j-1}\right)-F\left(t_{0}-S_{j}\right)}{F\left(t_{0}\right)}=\frac{\bar{F}\left(t_{0}-S_{j}\right)-\bar{F}\left(t_{0}-S_{j-1}\right)}{F\left(t_{0}\right)}=p \quad(j=1,2, \ldots, N) .
$$

Noting that $S_{0}=0$ and $S_{N}=t_{0}$, we easily have

$$
\bar{F}\left(t_{0}-S_{j}\right)=\bar{F}\left(t_{0}\right)+j p F\left(t_{0}\right) \quad(j=1,2, \ldots, N) .
$$

Thus, $S_{j}$ is given by the following function of $N$ :

$$
S_{j}=t_{0}-\bar{F}^{-1}\left[\bar{F}\left(t_{0}\right)+\frac{j}{N} F\left(t_{0}\right)\right]
$$

where $\bar{F}^{-1}$ is the inverse function of $\bar{F}$. Substituting $S_{j}$ into (8), we can obtain the total expected cost which is a function of $N$, and hence, we can easily derive an approximate optimal number $\widehat{N}$.

For example, when $\bar{F}(t)=\mathrm{e}^{-\lambda t}$, from (17),

$$
S_{j}=\frac{1}{\lambda} \log \left[1+\frac{j}{N}\left(\mathrm{e}^{\lambda t_{0}}-1\right)\right] \quad(j=1,2, \ldots, N),
$$

and the total expected cost is, from (8),

$$
\widehat{C}_{2}(N)=\frac{1}{N} \sum_{j=1}^{N}\left\{j c_{1}+\frac{c_{2}}{\lambda} \log \left[1+\frac{j}{N}\left(\mathrm{e}^{\lambda t_{0}}-1\right)\right]\right\} \quad(N=1,2, \ldots) .
$$




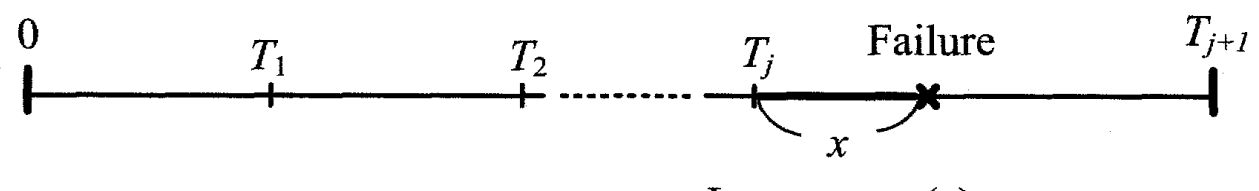

Loss $\operatorname{cost} c_{2}(x)$

Figure 3: Process of checking intervals

Therefore, we compute $\widehat{C}_{2}(N)$ for $N=1,2, \ldots$, and obtain an optimal $\widehat{N}$ which minimizes $\widehat{C}_{2}(N)$. Lastly, substituting $\widehat{N}$ in (19), we can obtain $\widehat{S}_{j}(j=1,2, \ldots, \widehat{N})$.

Table 4 presents $\widehat{C}_{2}(N) / c_{1}$ for $N=1,2, \ldots, 5$ and $\lambda t_{0}=1,2$ when $c_{2} /\left(\lambda c_{1}\right)=10$. When $\lambda t_{0}=1$ and 2 , we obtain optimal $\widehat{N}=3,4$, respectively. In particular, when $\lambda t_{0}=1$, $\widehat{N}=N^{*}$, and in this case, approximate backward times are, from (19), $\lambda \widehat{T}_{1}=0.453$, $\lambda \widehat{T}_{2}=0.311, \lambda \widehat{T}_{3}=0.236$, and the expected cost 9.387 is a little higher than 9.34 in Table 3.

\section{Backup Model}

The inspection models assume that failures can be only detected at the checking times using an inspection scheme in Barlow and Proschan [1, p. 107] and Nakagawa [7, p. 201]. We consider the case of a failure being detected in a database system, where a backup operation is performed to the last check point and the database is rebuilt from that point in Naruse et al. [14]. It is generally assumed in such models that any failure is always detected instantly, however, there is a loss time or cost associated with the elapsed time of the backup operation between the failure detection and the last check.

From the practical viewpoints of backup operation and database recovery, we take up three backup models by applying the inspection models. When the failure is detected, we execute the backup operation to the last check (Figure 3). The problem is to determine an optimal schedule of checking times which minimizes the expected cost.

\subsection{Periodic interval}

It is assumed that the failure time of the unit has a probability distribution $F(t)$ with a finite mean $\mu$ and a density function $f(t)$. The checking times are placed at periodic times $j T(j=1,2, \ldots)(0<T \leq \infty)$. Let $c_{1}$ be the cost for each check. In addition, when the failure is detected between $j T$ and $(j+1) T$, we execute the backup operation to the last checking time $j T$, which incurs a loss cost $c_{2}(x)$ (Figure 3).

The total expected cost until the backup operation is done to the last check when the unit has failed is, using the results of inspection policies in Barlow and Proschan [1, p. 108] and Nakagawa [7, p. 203],

$$
\begin{aligned}
C_{1}(T) & =\sum_{j=0}^{\infty} \int_{j T}^{(j+1) T}\left[c_{1} j+c_{2}(t-j T)\right] \mathrm{d} F(t) \\
& =\left[c_{1}-c_{2}(T)\right] \sum_{j=1}^{\infty} \bar{F}(j T)+\sum_{j=0}^{\infty} \int_{0}^{T} \bar{F}(t+j T) \mathrm{d} c_{2}(t)
\end{aligned}
$$

When $c_{2}(t)=c_{2} t$, the total expected cost is

$$
C_{1}(T)=\left(c_{1}-c_{2} T\right) \sum_{j=1}^{\infty} \bar{F}(j T)+c_{2} \mu
$$


Clearly,

$$
C_{1}(0) \equiv \lim _{T \rightarrow 0} C_{1}(T)=\infty, \quad C_{1}(\infty) \equiv \lim _{T \rightarrow \infty} C_{1}(T)=c_{2} \mu
$$

Hence,

$$
C_{1}(\infty)-C_{1}(T)=\left(c_{2} T-c_{1}\right) \sum_{j=1}^{\infty} \bar{F}(j T)
$$

Thus, there exits an optimal checking time $T^{*}\left(c_{1} / c_{2}<T^{*} \leq \infty\right)$ which minimizes $C_{1}(T)$ in (22).

Furthermore, differentiating $C_{1}(T)$ with respect to $T$ and setting it equal to zero,

$$
T-\frac{\sum_{j=1}^{\infty} \bar{F}(j T)}{\sum_{j=1}^{\infty} j f(j T)}=\frac{c_{1}}{c_{2}}
$$

which is a necessary condition for an optimum $T^{*}$ to satisfy. In the case of $F(t)=1-\mathrm{e}^{-\lambda t}$, (23) becomes

$$
T-\frac{1-\mathrm{e}^{-\lambda T}}{\lambda}=\frac{c_{1}}{c_{2}}
$$

whose left-hand side is strictly increasing from 0 to $\infty$. Thus, there exists a finite and unique $T^{*}\left(0<T^{*}<\infty\right)$ which satisfies $(24)$.

\subsection{Sequential interval}

Suppose that the checking times are placed at successive times $T_{j}(j=1,2, \ldots)$, where $T_{0} \equiv 0$. Then, by the similar method of obtaining (21), the total expected cost is

$$
\begin{aligned}
C_{2}(\mathrm{~T}) & =\sum_{j=0}^{\infty} \int_{T_{j}}^{T_{j+1}}\left[c_{1} j+c_{2}\left(t-T_{j}\right)\right] \mathrm{d} F(t) \\
& =\sum_{j=1}^{\infty}\left[c_{1}-c_{2}\left(T_{j}-T_{j-1}\right)\right] \bar{F}\left(T_{j}\right)+\sum_{j=0}^{\infty} \int_{0}^{T_{j+1}-T_{j}} \bar{F}\left(t+T_{j}\right) \mathrm{d} c_{2}(t)
\end{aligned}
$$

When $c_{2}(t)=c_{2} t$, the total expected cost is

$$
C_{2}(\mathbf{T})=\sum_{j=1}^{\infty}\left[c_{1}-c_{2}\left(T_{j}-T_{j-1}\right)\right] \bar{F}\left(T_{j}\right)+c_{2} \mu
$$

Differentiating $C_{2}(\mathbf{T})$ in (26) with respect to $T_{j}$ and setting it equal to zero,

$$
\frac{F\left(T_{j+1}\right)-F\left(T_{j}\right)}{f\left(T_{j}\right)}=T_{j}-T_{j-1}-\frac{c_{1}}{c_{2}} \quad(j=1,2, \ldots) .
$$

Thus, we can compute an optimal checking time $T_{j}^{*}$, using Algorithm 1 of Barlow and Proschan [1, p. 112]. Table 5 presents an optimal schedule $\left\{T_{j}^{*}\right\}$ which satisfies (27) when $F(t)=1-\exp \left[-(t / 500)^{2}\right], c_{1} / c_{2}=5,15,25$ and $\delta_{j} \equiv T_{j+1}^{*}-T_{j}^{*}$. The intervals $\delta_{j}$ between checking times increase with $c_{1} / c_{2}$ and decrease slowly with its number $j$. 
Table 5: Optimal checking times $T_{j}^{*}$ and $\delta_{j}=T_{j+1}^{*}-T_{j}^{*}$ when $F(t)=1-\exp \left[-(t / 500)^{2}\right]$

\begin{tabular}{c|cc|cc|cc}
\hline \multirow{2}{*}{$j$} & \multicolumn{6}{|c|}{$c_{1} / c_{2}$} \\
\cline { 2 - 7 } & $T_{j}^{*} 5$ & $\delta_{j}$ & $T_{j}^{*}$ & $\delta_{j}$ & $T_{j}^{*}$ & $\delta_{j}$ \\
\hline 1 & 113.87 & 83.81 & 165.71 & 122.70 & 197.96 & 147.31 \\
2 & 197.69 & 71.31 & 288.42 & 104.78 & 345.27 & 126.19 \\
3 & 269.00 & 63.95 & 393.20 & 94.22 & 471.46 & 113.73 \\
4 & 332.95 & 58.92 & 487.42 & 86.99 & 585.19 & 105.21 \\
5 & 391.87 & 55.18 & 574.41 & 81.61 & 690.41 & 98.88 \\
6 & 447.05 & 52.25 & 656.02 & 77.40 & 789.29 & 93.92 \\
7 & 499.29 & 49.87 & 733.42 & 73.97 & 883.21 & 89.88 \\
8 & 549.16 & 47.89 & 807.39 & 71.11 & 973.09 & 86.51 \\
9 & 597.05 & 46.20 & 878.50 & 68.66 & 1059.59 & 83.62 \\
10 & 643.26 & & 947.16 & & 1143.22 & \\
\hline
\end{tabular}

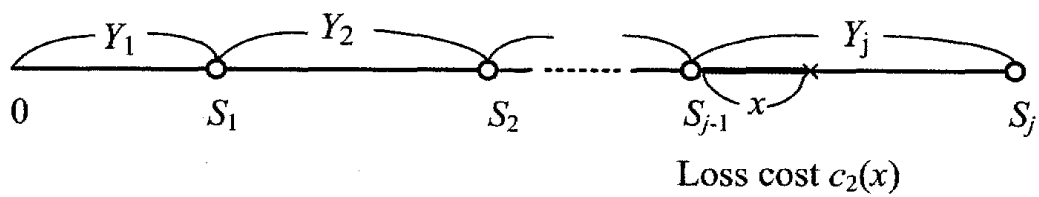

0 : Checking time $\quad \times$ : Failure

Figure 4: Process of checking times $Y_{j}$

\subsection{Random interval}

Most computer and database systems in offices and industries execute successively tasks, each of them has random working times. This section applies the backup operation to such systems: Checks are placed at every end of $N$ th $(N=1,2, \ldots)$ tasks. When the system fails, we execute the backup operation to the last check.

Suppose that task $j(j=1,2, \ldots)$ has a working time $Y_{j}$ with an identical distribution $G(t) \equiv \operatorname{Pr}\left\{Y_{j} \leq t\right\}$ with a finite mean $1 / \theta$, i.e., $1 / \theta=\int_{0}^{\infty} \bar{G}(t) \mathrm{d} t<\infty$. That is, tasks arrive successively at a renewal process with inter-arrival times $Y_{j}$, and the database system works for them according to a general distribution $G(t)$. Then, the probability that the system works exactly for $j$ tasks in $[0, t]$ is $G^{(j)}(t)-G^{(j+1)}(t)$, where $G^{(j)}(t)(j=1,2, \ldots)$ denotes the $j$-fold Stieltjes convolution of $G(t)$ with itself, i.e., $G^{(j)}(t) \equiv \int_{0}^{t} G^{(j-1)}(t-u) \mathrm{d} G(u)$ and $G^{(0)}(t) \equiv 1$ for $t \geq 0$. In addition, let $M(t)$ be a renewal function of $G(t)$, i.e., $M(t) \equiv$ $\sum_{j=1}^{\infty} G^{(j)}(t)$. The system fails with time $t$ according to a distribution $F(t)$, irrespective of the number of working tasks.

Suppose that the checking schedule of the system is done at the completion of successive working times $Y_{j}(j=1,2, \ldots)$, i.e., at times $S_{j} \equiv \sum_{i=1}^{j} Y_{i}(j=1,2, \ldots)$. Let $c_{1}$ be the cost required for each check. In addition, when the system fails between $S_{j-1}$ and $S_{j}$, we carry out the backup operation to the last checking time $S_{j-1}$. This incurs a loss cost $c_{2}(x)$ (Figure 4). Then, because the expected cost when the system fails in the working period of task $j$ is

$$
\int_{0}^{\infty}\left\{\int_{0}^{t}\left[c_{1}(j-1)+c_{2}(t-x)\right] \bar{G}(t-x) \mathrm{d} G^{(j-1)}(x)\right\} \mathrm{d} F(t)
$$


the total expected cost is

$$
\begin{aligned}
C_{3}(1) & =\sum_{j=1}^{\infty} \int_{0}^{\infty}\left\{\int_{0}^{t}\left[c_{1}(j-1)+c_{2}(t-x)\right] \bar{G}(t-x) \mathrm{d} G^{(j-1)}(x)\right\} \mathrm{d} F(t) \\
& \left.=c_{1} \int_{0}^{\infty} M(t) \mathrm{d} F(t)+\int_{0}^{\infty}\left[\int_{x}^{\infty} c_{2}(t-x)\right] \bar{G}(t-x) \mathrm{d} F(t)\right] \mathrm{d} M(x) .
\end{aligned}
$$

Next, suppose that the system is checked only at every $N$ th $(N=1,2, \ldots)$ working time, i.e., at the completion of the $j N$ working time. When $N=1$, the system is checked at every time of $S_{j}(j=1,2 \ldots)$. When $N=2$, i.e., the system is checked at times of $S_{2}$, $S_{4}, \ldots$, the total expected cost is

$$
\begin{aligned}
C_{3}(2) & =\sum_{j=1}^{\infty} \int_{0}^{\infty}\left\{\int_{0}^{t}\left[c_{1}(j-1)+c_{2}(t-x)\right] \bar{G}^{(2)}(t-x) \mathrm{d} G^{(2(j-1))}(x)\right\} \mathrm{d} F(t) \\
& \left.=c_{1} \int_{0}^{\infty} M^{(2)}(t) \mathrm{d} F(t)+\int_{0}^{\infty}\left[c_{2}(t) \bar{G}^{(2)}(t)+\int_{0}^{t} c_{2}(t-x)\right] \bar{G}^{(2)}(t-x) \mathrm{d} M^{(2)}(x)\right] \mathrm{d} F(t) .
\end{aligned}
$$

Generally, when the system is checked at the $j N$ th $(j=1,2, \ldots)$ working time, the total expected cost is

$$
\begin{aligned}
C_{3}(N) & =c_{1} \int_{0}^{\infty} M^{(N)}(t) \mathrm{d} F(t) \\
& \left.+\int_{0}^{\infty}\left[c_{2}(t) \bar{G}^{(N)}(t)+\int_{0}^{t} c_{2}(t-x)\right] \bar{G}^{(N)}(t-x) \mathrm{d} M^{(N)}(x)\right] \mathrm{d} F(t) \quad(N=1,2, \ldots)
\end{aligned}
$$

where $M^{(N)}(t) \equiv \sum_{j=1}^{\infty} G^{(j N)}(t)(N=1,2, \ldots)$. When $F(t)=1-\mathrm{e}^{-\lambda t}$ and $G(t)=1-\mathrm{e}^{-\theta t}$,

$$
\begin{aligned}
G^{(N)}(t) & =1-\sum_{j=0}^{N-1} \frac{(\theta t)^{j}}{j !} \mathrm{e}^{-\theta t} \\
\int_{0}^{\infty} \mathrm{e}^{-s t} \mathrm{~d} G^{(N)}(t) & =\left(\frac{\theta}{s+\theta}\right)^{N}, \\
\int_{0}^{\infty} \mathrm{e}^{-s t} \mathrm{~d} M^{(N)}(t) & =\sum_{j=1}^{\infty}\left[\left(\frac{\theta}{s+\theta}\right)^{N}\right]^{j}=\frac{\left(\frac{\theta}{s+\theta}\right)^{N}}{1-\left(\frac{\theta}{s+\theta}\right)^{N}}
\end{aligned}
$$

Thus, when $c_{2}(t)=c_{2} t$, the total expected cost is, from (30),

$$
C_{3}(N)=\frac{c_{1} A^{N}}{1-A^{N}}+\frac{c_{2} \lambda}{\theta(\theta+\lambda)} \frac{1}{1-A^{N}} \sum_{j=1}^{N} j A^{j} \quad(N=1,2, \ldots)
$$

where $A \equiv \theta /(\theta+\lambda)$.

From the inequality $C_{3}(N+1)-C_{3}(N) \geq 0$,

$$
\sum_{j=1}^{N}\left(1-A^{j}\right) \geq \frac{c_{1}(\theta+\lambda)}{c_{2}}
$$


Table 6: Values of $\sum_{j=1}^{N}\left(1-A^{j}\right)$

\begin{tabular}{c|cccccccc}
\hline & \multicolumn{8}{|c}{$\theta / \lambda$} \\
\cline { 2 - 9 }$N$ & 1 & 2 & 5 & 10 & 15 & 20 & 50 & 100 \\
\hline 1 & 0.50 & 0.33 & 0.17 & 0.09 & 0.06 & 0.057 & 0.02 & 0.01 \\
2 & 1.25 & 0.89 & 0.47 & 0.26 & 0.18 & 0.14 & 0.06 & 0.03 \\
3 & 2.13 & 1.59 & 0.89 & 0.51 & 0.36 & 0.28 & 0.12 & 0.06 \\
4 & 3.06 & 2.40 & 1.41 & 0.83 & 0.59 & 0.45 & 0.19 & 0.10 \\
5 & 4.03 & 3.26 & 2.01 & 1.21 & 0.86 & 0.67 & 0.29 & 0.15 \\
6 & 5.02 & 4.18 & 2.67 & 1.64 & 1.18 & 0.92 & 0.40 & 0.20 \\
7 & 6.01 & 5.12 & 3.40 & 2.13 & 1.55 & 1.21 & 0.53 & 0.27 \\
8 & 7.00 & 6.08 & 4.16 & 2.67 & 1.95 & 1.54 & 0.67 & 0.35 \\
9 & 8.00 & 7.05 & 4.97 & 3.24 & 2.39 & 1.89 & 0.84 & 0.43 \\
10 & 9.00 & 8.03 & 5.81 & 3.86 & 2.87 & 2.28 & 1.02 & 0.53 \\
\hline
\end{tabular}

whose left-hand side increases strictly from $\lambda /(\theta+\lambda)$ to $\infty$. Therefore, there exists a finite and unique minimum $N^{*}\left(1 \leq N^{*}<\infty\right)$ which satisfies (32). In particular, when $\lambda /(\theta+\lambda)^{2} \geq c_{1} / c_{2}$ then $N^{*}=1$.

Table 6 presents the values of $\sum_{j=1}^{N} A^{j}$ for $\theta / \lambda$ and $N$. For example, when $\theta / \lambda=10$, $A=10 / 11=0.91$ and if $0.09<c_{1}(\theta+\lambda) / c_{2} \leq 0.26$, then $N^{*}=1$, and if $0.26<c_{1}(\theta+\lambda) / c_{2} \leq$ 0.51 , then $N^{*}=2$, and so on.

\section{Traceability Model}

We apply the techniques of backward and backup to the traceability problem used commonly in food products and sales studied in Lees [6]: In this section, we propose four reliability models considering traceability and discuss optimal maintenance policies. Furthermore, we compare each model with and without traceability. Throughout this section, it is assumed that an operating unit has a failure distribution $F(t)$ with a finite mean $\mu<\infty$ and $\bar{F}(t) \equiv$ $1-F(t)$.

\subsection{Standard model}

Suppose that the unit begins to operate at time 0 and fails at time $t$, and its failure is detected at time $T(0<t \leq T)$. Then, we give the expected cost until failure detection as follows: For the unit with traceability,

$$
\begin{aligned}
C_{1}(T) & =c_{0}(T)+c_{1}+\int_{0}^{T} c_{2}(T-t) \mathrm{d} F(t) \\
& =c_{0}(T)+c_{1}+\int_{0}^{T} F(T-t) \mathrm{d} c_{2}(t)
\end{aligned}
$$

and for the unit without traceability

$$
\begin{aligned}
\bar{C}_{1}(T) & =c_{1}+\int_{0}^{T} c_{3}(T-t) \mathrm{d} F(t) \\
& =c_{1}+\int_{0}^{T} F(T-t) \mathrm{d} c_{3}(t),
\end{aligned}
$$

where $c_{0}(T)$ is the tracing cost during $(0, T], c_{1}$ is the cost of one check, and $c_{2}(x)$ and $c_{3}(x)$ are the loss cost of searching for the actual failure time of the unit with and without 
traceability, respectively, when the failure is detected at time $T$. It would be generally shown in practical fields that $c_{2}(x)<c_{3}(x)$, because it would be much easier to find the failure time by following up the trace than no trace.

First, when $c_{i}(t)=c_{i} t(i=0,2,3)$, the expected cost rate is given by

$$
\widetilde{C}_{1}(T) \equiv \frac{C_{1}(T)}{T}=c_{0}+\frac{c_{1}+c_{2} \int_{0}^{T} F(t) \mathrm{d} t}{T} .
$$

We find an optimal $T_{1}^{*}$ which minimizes $\widetilde{C}_{1}(T)$. Clearly, because

$$
\lim _{T \rightarrow 0} \widetilde{C}_{1}(T)=\infty, \quad \lim _{T \rightarrow \infty} \widetilde{C}_{1}(T)=c_{0}+c_{2}
$$

there exists a positive $T_{1}^{*}\left(0<T_{1}^{*} \leq \infty\right)$. Differentiating $\widetilde{C}_{1}(T)$ with respect to $T$ and setting it equal to zero,

$$
\int_{0}^{T} t \mathrm{~d} F(t)=\frac{c_{1}}{c_{2}}
$$

Therefore, if $c_{2} \mu>c_{1}$, then there exists a finite and unique $T_{1}^{*}\left(0<T_{1}^{*}<\infty\right)$ which satisfies (36).

Next, compare the expected cost $C_{1}(T)$ in $(33)$ and $\bar{C}_{1}(T)$ in (34) for $c_{3}>c_{2}$. From the inequality $\bar{C}_{1}(T)>C_{1}(T)$

$$
\frac{1}{T} \int_{0}^{T} F(t) \mathrm{d} t>\frac{c_{0}}{c_{3}-c_{2}}
$$

whose left-hand side is strictly increasing from 0 to 1 . Thus, if $c_{3}>c_{2}+c_{0}$, then there exists a finite and unique $\widetilde{T}_{1}$ which satisfies

$$
\frac{1}{T} \int_{0}^{T} F(t) \mathrm{d} t=\frac{c_{0}}{c_{3}-c_{2}} .
$$

Therefore, if $c_{3}>c_{2}+c_{0}$ and $T>\widetilde{T}_{1}$, then $\bar{C}_{1}(T)>C_{1}(T)$, i.e., we should trace the unit.

In particular, when the failure time is exponential, i.e., $F(t)=1-\mathrm{e}^{-\lambda t}$, from (36), if $c_{2} / \lambda>c_{1}$, then there exists a finite and unique $T_{1}^{*}\left(0<T_{1}^{*}<\infty\right)$ which satisfies

$$
1-(1+\lambda T) \mathrm{e}^{-\lambda T}=\frac{c_{1}}{c_{2} / \lambda} \text {. }
$$

Furthermore, because $1-\mathrm{e}^{-a}<a$ for $a>0$,

$$
T_{1}^{*}>\sqrt{\frac{c_{1}}{c_{2} \lambda}}
$$

Next, when $c_{3}>c_{2}+c_{0}$, from (38), there exists a finite and unique $\widetilde{T}_{1}$ which satisfies

$$
1-\frac{1-\mathrm{e}^{-\lambda T}}{\lambda T}=\frac{c_{0}}{c_{3}-c_{2}}
$$

In this case, if $T>\widetilde{T}_{1}$, then we should trace the unit, and

$$
\lambda \widetilde{T}_{1}>\frac{2 c_{0}}{c_{3}-c_{2}} .
$$

Table 7 presents optimal time $\lambda T_{1}^{*}$ for $\left(c_{2} / \lambda\right) / c_{1}$ and boundary time $\lambda \widetilde{T}_{1}$ for $\left(c_{3}-c_{2}\right) / c_{0}$. For example, when $c_{2} /\left(c_{1} \lambda\right)=5$ and the failure is checked and detected at time $\lambda T_{1}^{*}=0.8244$, if $\left(c_{3}-c_{2}\right) / c_{0} \leq 3$ then $\lambda T_{1}^{*}<\lambda \widetilde{T}_{1}=0.8742$, and hence, we should not trace the unit, and if $\left(c_{3}-c_{2}\right) / c_{0} \geq 4$, we should do it. When $\left(c_{3}-c_{2}\right) / c_{0}=10$, if $c_{2} /\left(c_{1} \lambda\right) \leq 50$ then $\lambda T_{1}^{*}>\lambda \widetilde{T}_{1}$ and we should trace the unit. 
Table 7: Optimal time $\lambda T_{1}^{*}$ and boundary time $\lambda \widetilde{T}_{1}$

\begin{tabular}{c|c|c|c}
\hline$\frac{c_{2} / \lambda}{c_{1}}$ & $\lambda T_{1}^{*}$ & $\frac{c_{3}-c_{2}}{c_{0}}$ & $\lambda \widetilde{T}_{1}$ \\
\hline 2 & 1.6783 & 2 & 1.5936 \\
3 & 1.1888 & 3 & 0.8742 \\
4 & 0.9613 & 4 & 0.6059 \\
5 & 0.8244 & 5 & 0.4642 \\
10 & 0.5318 & 6 & 0.3764 \\
20 & 0.3554 & 7 & 0.3166 \\
30 & 0.2835 & 8 & 0.2733 \\
50 & 0.2147 & 9 & 0.2404 \\
60 & 0.1947 & 10 & 0.2146 \\
\hline
\end{tabular}

\subsection{Finite interval model}

The unit has to operate for a finite interval $[0, T]$. Then, we give the expected cost for this interval as follows: For the unit with traceability,

$$
C_{2}(T)=c_{0}(T)+\int_{0}^{T} c_{2}(T-t) \mathrm{d} F(t)
$$

and for the unit without traceability,

$$
\bar{C}_{2}(T)=\int_{0}^{T}\left[c_{1}(T)+c_{3}(T-t)\right] \mathrm{d} F(t)
$$

where $c_{1}(T)$ is the additional tracing cost during $[0, T]$ of the unit without traceability when the failure is detected at time $T$. When $c_{i}(t)=c_{i} t(i=0,1,2,3)$, from the inequality $\bar{C}_{2}(T)>C_{2}(T)$

$$
c_{1} F(T)+\frac{c_{3}-c_{2}}{T} \int_{0}^{T} F(t) \mathrm{d} t>c_{0},
$$

whose left-hand side is strictly increasing from 0 to $c_{1}+c_{3}-c_{2}$. Thus, if $c_{1}+c_{3}>c_{2}+c_{0}$, then there exists a finite and unique $\widetilde{T}_{2}$ which satisfies

$$
c_{1} F(T)+\frac{c_{3}-c_{2}}{T} \int_{0}^{T} F(t) \mathrm{d} t=c_{0} .
$$

Therefore, if $c_{1}+c_{3}>c_{2}+c_{0}$ and $T>\widetilde{T}_{2}$, then we should trace the unit for the interval $[0, T]$.

\subsection{Periodic model}

Suppose that the unit is checked only at periodic times $j T(j=1,2, \ldots)$ and the failure is detected at the next check. Then, we give the expected cost until failure detection as follows: For the unit with traceability,

$$
C_{3}(T)=\sum_{j=1}^{\infty} \int_{(j-1) T}^{j T}\left[c_{0}(j T)+c_{1} j+c_{2}(j T-t)\right] \mathrm{d} F(t),
$$

and for the unit without traceability

$$
\bar{C}_{3}(T)=\sum_{j=1}^{\infty} \int_{(j-1) T}^{j T}\left[c_{1} j+c_{3}(j T-t)\right] \mathrm{d} F(t),
$$


where $c_{1}$ is the cost of one check, and $c_{0}(t), c_{2}(t)$ and $c_{3}(t)$ are given in (33) and (34).

When $c_{i}(t)=c_{i} t(i=0,2,3)$, the expected cost is, from (47),

$$
C_{3}(T)=\left(c_{0} T+c_{1}+c_{2} T\right) \sum_{j=0}^{\infty} \bar{F}(j T)-c_{2} \mu
$$

and from (48),

$$
\bar{C}_{3}(T)=\left(c_{1}+c_{3} T\right) \sum_{j=0}^{\infty} \bar{F}(j T)-c_{3} \mu .
$$

We find an optimal $T_{3}^{*}$ which minimizes $C_{3}(T)$ in (49). Differentiating $C_{3}(T)$ with respect to $T$ and setting it equal to zero,

$$
\frac{\sum_{j=0}^{\infty} \bar{F}(j T)}{\sum_{j=1}^{\infty} j f(j T)}-T=\frac{c_{1}}{c_{0}+c_{2}},
$$

where $f(t)$ is a density function of $F(t)$. Thus, $T_{3}^{*}$ is given by a solution of (51).

Next, we compare $C_{3}(T)$ in (49) and $\bar{C}_{3}(T)$ in (50) for $c_{3}>c_{2}+c_{0}$. Then, from the inequality $\bar{C}_{3}(T)-C_{3}(T)>0$,

$$
\frac{T}{\mu} \sum_{j=0}^{\infty} \bar{F}(j T)>\frac{c_{3}-c_{2}}{c_{3}-c_{2}-c_{0}} .
$$

In particular, when $F(t)=1-\mathrm{e}^{-\lambda t}$, from (51), an optimal $T_{3}^{*}$ is given by a finite and unique solution of the equation

$$
\mathrm{e}^{\lambda T}-(1+\lambda T)=\frac{\lambda c_{1}}{c_{0}+c_{2}}
$$

Furthermore, from (52), if $c_{3}>c_{2}+c_{0}, \widetilde{T}_{3}$ is given by a finite and unique solution of the equation

$$
1-\frac{1-\mathrm{e}^{-\lambda T}}{\lambda T}=\frac{c_{0}}{c_{3}-c_{2}}
$$

In this case, if $c_{3}>c_{2}+c_{0}$ and $T_{3}^{*}>\widetilde{T}_{3}$, then we should trace the unit and make the periodic inspection at times $j T_{3}^{*}$.

\subsection{Sequential model}

Suppose that the unit is checked at successive times $T_{j}(j=1,2, \ldots)$, where $T_{0} \equiv 0$. By the similar method of obtaining the expected costs $C_{3}(T)$ and $\bar{C}_{3}(T)$ in Section 4.3 , the expected cost for the unit with traceability is

$$
\begin{aligned}
C_{4}(\mathbf{T}) & =\sum_{j=1}^{\infty} \int_{T_{j-1}}^{T_{j}}\left[c_{0}\left(T_{j}\right)+c_{1} j+c_{2}\left(T_{j}-t\right)\right] \mathrm{d} F(t) \\
& =\sum_{j=0}^{\infty}\left[c_{0}\left(T_{j+1}\right)-c_{0}\left(T_{j}\right)+c_{1}+c_{2}\left(T_{j+1}-T_{j}\right)\right] \bar{F}\left(T_{j}\right)+\sum_{j=1}^{\infty} \int_{T_{j-1}}^{T_{j}} \bar{F}(t) \mathrm{d} c_{2}\left(T_{j}-t\right),
\end{aligned}
$$


and for the unit without traceability

$$
\begin{aligned}
\bar{C}_{4}(\mathbf{T}) & =\sum_{j=1}^{\infty} \int_{T_{j-1}}^{T_{j}}\left[c_{1} j+c_{3}\left(T_{j}-t\right)\right] \mathrm{d} F(t) \\
& =\sum_{j=0}^{\infty}\left[c_{1}+c_{3}\left(T_{j+1}-T_{j}\right)\right] \bar{F}\left(T_{j}\right)+\sum_{j=1}^{\infty} \int_{T_{j-1}}^{T_{j}} \bar{F}(t) \mathrm{d} c_{3}\left(T_{j}-t\right)
\end{aligned}
$$

We find an optimal checking schedule which minimizes $C_{4}(\mathbf{T})$ in $(55)$ when $c_{i}(t)=c_{i} t$ $(i=0,2)$. In this case, the expected cost is

$$
C_{4}(\mathrm{~T})=\sum_{j=0}^{\infty}\left[\left(c_{0}+c_{2}\right)\left(T_{j+1}-T_{j}\right)+c_{1}\right] \bar{F}\left(T_{j}\right)-c_{2} \mu
$$

Differentiating $C_{4}(\mathbf{T})$ with respect to $T_{j}$ and setting it equal to zero,

$$
T_{j+1}-T_{j}=\frac{F\left(T_{j}\right)-F\left(T_{j-1}\right)}{f\left(T_{j}\right)}-\frac{c_{1}}{c_{0}+c_{2}} .
$$

Similarly, differentiating $\bar{C}_{4}(\mathbf{T})$ with respect to $T_{j}$ and setting it equal to zero when $c_{3}(t)=$ $c_{3} t$

$$
T_{j+1}-T_{j}=\frac{F\left(T_{j}\right)-F\left(T_{j-1}\right)}{f\left(T_{j}\right)}-\frac{c_{1}}{c_{3}} .
$$

It is noted that both (58) and (59) correspond to the type of the equation in Barlow and Proschan $\left[1\right.$, p. 110] and Nakagawa $\left[7\right.$, p. 203]. Thus, we can compute optimal times $T_{j}^{*}$ for specified $F(t)$ and cost $c_{i}(i=0,1,2,3)$, using Algorithm 1 of Barlow and Proschan $[1$, p. 112], by a similar method in Section 3.2 .

\section{Conclusions}

We have summarized a variety of the backward and backup models, and derived their optimal maintenance policies using maintenance and reliability theory. Furthermore, we have applied such models to the traceability problem used commonly in food products. Such concept of traceability would be applied practically to more production systems to lessen inferior goods and to recover them fast.

There exist many present situations in which we have to go back to some point and restore a normal condition as soon as possible by some suitable maintenance, when failures have occurred or been detected. There are a few papers treated with such backward models. This survey paper would be more useful for studying theoretically these problems and for applying practically to actual systems.

\section{References}

[1] R.E. Barlow and F. Proschan: Mathematical Theory of Reliability (John Wiley \& Sons, New York, 1965).

[2] N.K. Chandra and D. Roy: Some results on reversed hazard rate. Probability in Engineering and Information Science, 15 (2001), 95-102.

[3] M.S. Finkelstein: On the reversed hazard rate. Reliability Engineering and System Safety, 78 (2002), 71-75. 
[4] S. Fukumoto, N. Kaio and S. Osaki: A study of checkpoint generations for a database recovery mechanism. Computers and Mathematics, 24 (1992), 63-70.

[5] N. Kaio and S. Osaki: Comparison of inspection policies. Journal of Operational Research Society, 40 (1989), 499-503.

[6] M. Lees: Food Authenticity and Traceability (Woodhead Publishing Ltd., Cambridge, 2003).

[7] T. Nakagawa: Maintenance Theory of Reliability (Springer-Verlag, London, 2005).

[8] T. Nakagawa: Shock and Damage Models in Reliability Theory (Springer-Verlag, London, 2007).

[9] T. Nakagawa: Advanced Reliability Models and Maintenance Policies (Springer-Verlag, London, 2008).

[10] S. Nakagawa, S. Fukumoto and N. Ishii: Optimal checkpointing intervals of three error detection schemes by a double modular redundancy. Mathematical and Computing Modeling, 38 (2003), 1357-1363.

[11] T. Nakagawa and S. Mizutani: A summary of maintenance policies for a finite interval. Reliability Engineering and System Safety, 94 (2009), 89-96.

[12] T. Nakagawa and S. Mizutani: Optimum problems in backward times of reliability models. IIE Transactions, 41 (2009), 65-71.

[13] S. Nakamura, C.H. Qian, S. Fukumoto and T. Nakagawa: Optimal backup policy for a database system with incremental and full backups. Mathematical and Computing Modeling, 38 (2003), 1373-1379.

[14] K. Naruse, S. Nakagawa and Y. Okuda: Optimal checking time of backup operation for a database system. In T. Dohi, S. Osaki and K. Sawaki (eds.): Recent Advances in Stochastic Operations Research (World Scientific Publishing, Singapore, 2007),131-144.

[15] S. Osaki (ed.) Stochastic Models in Reliability and Maintenance. (Springer-Verlag, Berlin, 2002).

[16] H. Pham: Handbook of Reliability Engineering. (Springer-Verlag, London, 2003).

[17] C.H. Qian, Y. Pan and T. Nakagawa: Optimal policies for a database system with two backup schemes. RAIRO Operations Research, 36 (2002), 227-235.

[18] A. Reuter: Performance analysis of recovery techniques. ACM Transactions on Database Systems, 9 (1984), 526-559.

[19] H. Sandoh and N. Igaki: Inspection policies for a scale. Journal of Quality in Maintenance Engineering, 7 (2001), 220-231.

[20] H. Sandoh and T. Nakagawa: How much should we reweigh?. Journal of Operational Research Society, 54 (2003), 318-321.

[21] T. Sugiura, S. Mizutani and T. Nakagawa: Optimal random and periodic inspection policies. In H. Pham (ed.): Probability Modeling, Analysis and Optimization (World Scientific Publishing, Singapore, 2006), 393-403.

[22] R. Velpuri and A. Adkoli: Oracle 8 Backup and Recovery Handbook. (McGraw-Hill, England, 2000). 
Mingchih Chen

Department of Industrial Engineering and Management

Chaoyang University of Technology 168 Jifong E. Road, Wufong Township 41349, Taiwan, R.O.C.

E-mail: mchen@cyut.edu.tw 